\section{Wrong word too early}

Invertebrate Endocrinology and Hormonal Heterophylly. Edited by Walter J. Burdette. Pp. xviii +438. (Springer : Berlin and New York, 1974.) \$21.50.

Heterophylly, as defined by the Oxford Dictionary, is a botanical term referring to plants having leaves of more than one shape. How then does this relate to invertebrate hormones? In fact, it does not. The editor has given a new meaning to the word, and uses it to describe the effects of hormones from one phylum on individuals of another phylum. From the title one expects to read about hormones from various invertebrate groups. But, in truth, the book considers only insect hormones, and of these, only ecdysone, juvenile hormone, and brain hormone.

The book contains contributions made to a meeting held at Houston early in 1971. Unfortunately, there has been a long delay between the submission of the manuscripts and the appearance of the book in late 1974, and, consequently, it is rare to find a reference later than 1970; in fast moving areas of research, such as juvenile hormone and ecdysone biosynthesis and degradation, many papers are already out of date.

The book is divided into four parts. Part I, "Invertebrate endocrinology", deals with the control of metamorphosis, hormone assays, and hormone biosynthesis and degradation. The section starts promisingly with remarks by Wigglesworth on the use of hormones to probe basic problems in the regulation of growth and differentiation. The following papers include articles that cater to a general audience as well as those with very technical information aimed at the specialist. The series of papers on assay techniques, an example of the latter, are varied in their usefulness. The paper by Bjerke and Roller on juvenile hormone assays provides a broad, critical comparison of the strengths and weaknesses of the common bioassay systems that were in use at the time. By contrast, the treatments of the brain hormone and ecdysone assays are much more limited in their scope and, accordingly, are of less value.

The second part considers primarily the isolation and distribution of various ecdysones. Since the original report in 1966 by Nakanishi and his coworkers of the presence of ecdysone-like chemicals in plants, the isolation and identification of these substances has, with a few notable exceptions, been carried out by workers in Japan. The articles in this section serve the valuable function of bringing together the data from the Japanese literature on the structure of the known phytoecyd- sones ( 37 at the time the paper was written) as well as extensive listings of their plant sources.

Part III comprises six papers on the effects of vertebrate hormones on vertebrate tissues. Except for presumed parallels between the mode of action of these hormones on vertebrates, and that of insect hormones on insects, these papers bear little relationship to the rest of the book and seem to be quite out of place.

Hormonal heterophylly is the subject of the last part. From the various articles, it quickly becomes apparent that the effects of insect hormones on

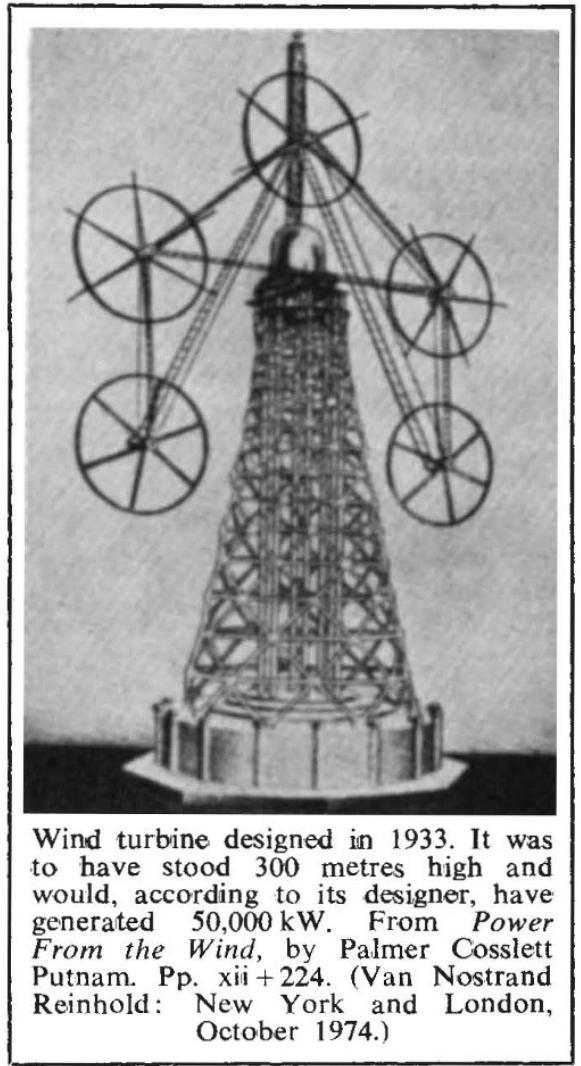

vertebrate cells are neither striking nor profound. The effects of ecdysone on the growth of mammalian tumours and on human leucocyte chromosomes are at best slight and are certainly of questionable statistical significance. The insect hormones do cause metabolic changes in mouse liver, but this can scarcely be surprising in a tissue adapted for detoxification of foreign substances. Indeed, the fact that insect hormones have little effect on vertebrate tissues, as documented by the papers dealing with pharmacological screens of these hormones, is a point in their favour. Especially as one day they may be released into the environment as insecticides.

The editor of this volume states that it is the first concerted effort to collect and analyse responses of vertebrate, as well as invertebrate, tissues to insect hormones. In my opinion, it is a premature attempt. James W. Truman

\section{Old bones}

Bones for the Archaeologist. By I. W. Cornwall. Revised edition. Pp. 259. (Dent: London, February 1975.) £4.50.

THIs is a revised edition of a volume first published in 1956. The section on conservation has some useful additions, the bibliography and the references have been improved and brought up to date and a very necessary index has been added. The bulk of the text and the illustrations remain the same.

When this book was first published the intention was to help the archaeologist make preliminary identifications of bones from archaeological sites at a time when much data had accumulated in museums awaiting the interest of some specialists from the natural sciences. It demonstrated successfully that most of this work could be done by the archaeologist himself if he was prepared to apply himself to learning the basic necessities of bone analysis. Many archaeologists, who were not entirely artefactually orientated, found it both necessary and relatively easy to add to their competence. There can be little doubt that some improvement in excavation techniques was one of the consequences of the publication of their book, as well as a reduction in the backlog of material awaiting analysis and identification.

The volume contains chapters on zoological classification and nomenclature, bone identification, field work, laboratory work and conservation, the estimation of age, sex and stature, and study and interpetation. For the tiro it even suggests logical methods of procedure. It is easily the best introductory work available at the present time and will guide many newcomers to archaeology to a useful and essentially practical expertise.

On the other hand, the weakest section is that concerned with study and interpretation. There is no clear indication of what purposes underlie the collection of bone specimens nor, in consequence, how bones should be collected on the site to give an adequate sample. It still remains that, in contrast to pollen analysis, data is atrociously collected during excavation by archaeologists with vastly different interests, techniques, knowledge and relevant expertise; there is still no discipline about archaeological collection. In consequence, samples obtained are commonly of minimal value and amenable to valid interpretation only at the coarsest levels. This fact could have been pointed out to advantage at a time when numerical analyses are increasingly fashionable. Excellent teaching as, for the most part, it is, Dr Cornwall's book stays with the vision of some 20 years ago.

E. S. Higgs 